\title{
Dynamics of anticorrelation effects in Raman scattering by phonons
}

\author{
P Szlachetka $\dagger$, S Kielich $\dagger$, J Peřina $\$$ and V Peřinováł \\ $\uparrow$ Nonlinear Optics Division, Institute of Physics, A. Mickiewicz University, Grunwaldzka \\ 6, 60-780 Poznań, Poland \\ * Laboratory of Optics, Palacký University, Leninova 26, 77146 Olomouc, Czechoslovakia
}

Received 18 November 1978, in final form 23 January 1979

\begin{abstract}
Photon-photon and photon-phonon anticorrelation effects in Raman scattering are studied theoretically. The Heisenberg equations of motion describing Raman scattering are solved in the short-time approximation. The laser, Stokes, anti-Stokes and phonon modes are quantised. The joint normally ordered characteristic functions are evaluated for modes exhibiting anticorrelation effects. The problem of existence of a Glauber-Sudarshan quasi-distribution function related to anticorrelation effects is considered. The magnitude of the anticorrelation effects depends on the initial statistical properties of the photon and phonon modes.
\end{abstract}

\section{Introduction}

It has been known for some time (Shen 1967, Walls 1973, Simaan 1975) that the statistical properties of Stokes and anti-Stokes fields depend strongly on the initial statistical nature of the photons and phonons. The purpose of this paper is to consider anticorrelation effects in Raman scattering by phonons from a quantum statistical point of view, and to answer the question of whether and how the anticorrelation effects depend on the initial statistical properties of the photons and phonons.

Anticorrelation effects can occur in individual modes as well as between different modes. In the first case, the variance of the number of photons is less than the average number of photons (or equivalently, the variance of intensity is negative) and the photo-counting distribution becomes narrower than the corresponding Poisson distribution for a coherent state (Peřina 1972). In the anticorrelation effect occurring between two different modes, the covariance function for the number of photons is negative. Anticorrelation effects have been studied for parametric amplification processes (Stoler 1974, Mišta and Peřina 1977), second-harmonic generation (Kozierowski and Tanaś 1977, Kielich et 1978, Peřinová and Peřina 1978, Mostowski and Rzążewski 1978, Drummond et al 1979), two-photon absorption (Simaan and Loudon 1975, Bandilla and Ritze 1976, Chatarvedi et al 1977) and two-photon stimulated emission (Yuen 1976). With regard to resonance fluorescence, the prediction of photon antibunching was made by Carmichael and Walls (1976), and photon anticorrelation was detected experimentally by Kimble et al (1977). In the present paper we consider the possible existence of anticorrelation effects in stimulated and spontaneous Raman scattering between the incident laser and scattered field (Stokes and anti-Stokes radiation) and between photons and phonons. In our investigation, we 
apply the model Hamiltonian for Raman scattering suggested by Mollow and Glauber (1967) and extended to the trilinear case by Walls (1970). The Heisenberg equations of motion describing the dynamics of the Raman scattering are solved in the short-time approximation. In our treatment all fields-laser, Stokes, anti-Stokes and phononare quantized. The molecular vibrations are described by chaotic phonons. The incident laser field is coherent. We consider the Stokes and anti-Stokes fields as being initially coherent, or chaotic. Using the solution of the Heisenberg equations of motion we evaluate the quantum normally ordered characteristic functions. With the help of these functions, we calculate the variances of intensity and covariance functions for the photons and phonons. We consider the existence problem of normally ordered quasi-distribution functions.

\section{Hamiltonian formulation of Raman scattering by phonons}

The single act of Raman scattering by phonons may be described as follows. A laser photon with frequency $\omega_{1}$ incident on a Raman active medium is annihilated, and a phonon with frequency $\omega_{\mathrm{p}}$ and a Stokes photon with frequency $\omega_{\mathrm{S}}=\omega_{1}-\omega_{\mathrm{p}}$ are created. Alternatively, an incident laser photon together with a phonon may be annihilated and create an anti-Stokes photon with the frequency $\omega_{\mathrm{a}}=\omega_{1}+\omega_{\mathrm{p}}$. Therefore, the model Hamiltonian describing the above Raman scattering has the form (Walls 1970, 1973, von Forester and Glauber 1971)

$$
\begin{aligned}
& \hat{H}=\hat{H}_{0}+\hat{H}_{\mathrm{S}}+\hat{H}_{\mathrm{a}} \\
& \hat{H}_{0}=\hbar \omega_{1} \hat{a}_{l}^{+} \hat{a}_{1}+\hbar \omega_{\mathrm{p}} \hat{a}_{\mathrm{p}}^{+} \hat{a}_{\mathrm{p}}+\hbar \omega_{\mathrm{s}} \hat{a}_{\mathrm{s}}^{+} \hat{a}_{\mathrm{S}}+\hbar \omega_{\mathrm{a}} \hat{a}_{\mathrm{a}}^{+} \hat{a}_{\mathrm{a}} \\
& \hat{H}_{\mathrm{s}}=\hbar\left(\mathscr{H}_{\mathrm{s}} \hat{a}_{1} \hat{a}_{\mathrm{p}}^{+} \hat{a}_{\mathrm{S}}^{+}+\mathrm{HC}\right) \\
& \hat{H}_{\mathrm{a}}=\hbar\left(\mathscr{H}_{\mathrm{a}} \hat{a}_{1} \hat{a}_{\mathrm{p}} \hat{a}_{\mathrm{a}}^{+}+\mathrm{HC}\right)
\end{aligned}
$$

where $\omega_{1}, \hat{a}_{1}^{+}, \hat{a}_{1} ; \omega_{\mathrm{s}}, \hat{a}_{\mathrm{s}}^{+}, \hat{a}_{\mathrm{s}} ; \omega_{\mathrm{a}}, \hat{a}_{\mathrm{a}}^{+}, \hat{a}_{\mathrm{a}}$ and $\omega_{\mathrm{p}}, \hat{a}_{\mathrm{p}}^{+}, \hat{a}_{\mathrm{p}}$ denote the frequencies and the creation and annihilation operators for laser, Stokes, anti-Stokes and phonon modes respectively. The Stokes coupling constant $\mathscr{H}_{S}$ and anti-Stokes coupling constant $\mathscr{H}_{\text {a }}$ contain the phase integrals

$$
\int_{v} \exp \left[-\mathrm{i}\left(\boldsymbol{k}_{1}-\boldsymbol{k}_{\mathrm{p}}-\boldsymbol{k}_{\mathrm{s}}\right) \cdot \boldsymbol{r}\right] \mathrm{d}^{3} r
$$

and

$$
\int_{v} \exp \left[-\mathrm{i}\left(k_{1}+k_{\mathrm{p}}-k_{\mathrm{a}}\right) \cdot r\right] \mathrm{d}^{3} r
$$

respectively, where $\boldsymbol{k}_{\mathrm{j}}$ are the wavevectors of the modes. The dynamics of the system described by the model Hamiltonian (2.1) are given by the following equations of motion in the Heisenberg picture:

$$
\begin{aligned}
& \mathrm{d} \hat{a}_{1}(t) / \mathrm{d} t=-\mathrm{i} \omega_{1} \hat{a}_{1}(t)-\mathrm{i} \mathscr{H}_{\mathrm{S}}^{*} \hat{a}_{\mathrm{p}}(t) \hat{a}_{\mathrm{S}}(t)-\mathrm{i} \mathscr{H}_{\mathrm{a}}^{*} \hat{a}_{\mathrm{p}}^{+}(t) \hat{a}_{\mathrm{a}}(t) \\
& \mathrm{d} \hat{a}_{\mathrm{p}}(t) / \mathrm{d} t=-\mathrm{i} \omega_{\mathrm{p}} \hat{a}_{\mathrm{p}}(t)-\mathrm{i} \mathscr{H}_{\mathrm{S}} \hat{a}_{1}(t) \hat{a}_{\mathrm{S}}^{+}(t)-\mathrm{i} \mathscr{H}_{\mathrm{a}}^{*} \hat{a}_{1}^{+}(t) \hat{a}_{\mathrm{a}}(t) \\
& \mathrm{d} \hat{a}_{\mathrm{S}}(t) / \mathrm{d} t=-\mathrm{i} \omega_{\mathrm{s}} \hat{a}_{\mathrm{S}}(t)-\mathrm{i} \mathscr{H}_{\mathrm{S}} \hat{a}_{1}(t) \hat{a}_{\mathrm{p}}^{+}(t) \\
& \mathrm{d} \hat{a}_{\mathrm{a}}(t) / \mathrm{d} t=-\mathrm{i} \omega_{\mathrm{a}} \hat{a}_{\mathrm{a}}(t)-\mathrm{i} \mathscr{H}_{\mathrm{a}} \hat{a}_{1}(t) \hat{a}_{\mathrm{p}}(t) .
\end{aligned}
$$


Denoting the number operators $\hat{a}_{1}^{+}(t) \hat{a}_{1}(t), \hat{a}_{\mathrm{S}}^{+}(t) \hat{a}_{\mathrm{S}}(t), \hat{a}_{\mathrm{a}}^{+}(t) \hat{a}_{\mathrm{a}}(t)$ and $\hat{a}_{\mathrm{p}}^{+}(t) \hat{a}_{\mathrm{p}}(t)$ by $\hat{n}_{\mathrm{I}}(t), \hat{n}_{\mathrm{S}}(t), \hat{n}_{\mathrm{a}}(t)$ and $\hat{n}_{\mathrm{p}}(t)$ respectively, we may verify that the conservation laws for the number operators are given by

$$
\begin{aligned}
& (\mathrm{d} / \mathrm{d} t)\left(\hat{n}_{1}(t)+\hat{n}_{\mathrm{S}}(t)+\hat{n}_{\mathrm{a}}(t)\right)=0 \\
& (\mathrm{~d} / \mathrm{d} t)\left(\hat{n}_{\mathrm{p}}(t)+\hat{n}_{\mathrm{a}}(t)-\hat{n}_{\mathrm{S}}(t)\right)=0 .
\end{aligned}
$$

Thus we find that $\hat{n}_{1}(t)+\hat{n}_{\mathrm{S}}(t)+\hat{n}_{\mathrm{a}}(t)$ and $\hat{n}_{\mathrm{p}}(t)+\hat{n}_{\mathrm{a}}(t)-\hat{n}_{\mathrm{S}}(t)$ are constants of motion in the Raman effect and are simultaneously satisfied for any time. The Heisenberg equations of motion (2.5)-(2.8) are coupled nonlinear equations. In order to solve them, we had recourse to the short-time technique described and applied by Agrawal and Mehta (1974) and Gambini (1977) to study the dynamics of parametric processes with a trilinear Hamiltonian. Using the short-time technique we can write an approximate solution (up to second order in $t$ ) of the Heisenberg equations $(2.5)-(2.8)$ in the form

$$
\begin{aligned}
& \hat{a}_{1}(t)=\left[\hat{a}_{1}-\mathrm{i} \mathscr{H}_{\mathrm{S}}^{*} t \hat{a}_{\mathrm{p}} \hat{a}_{\mathrm{S}}-\mathrm{i} \mathscr{H}_{\mathrm{a}}^{*} t \hat{a}_{\mathrm{p}}^{+} \hat{a}_{\mathrm{a}}-\frac{1}{2}\left|\mathscr{H}_{\mathrm{S}}\right|^{2} t^{2} \hat{a}_{1}\left(\hat{a}_{\mathrm{S}}^{+} \hat{a}_{\mathrm{s}}+\hat{a}_{\mathrm{p}}^{+} \hat{a}_{\mathrm{p}}+1\right)\right. \\
& \left.+\frac{1}{2}\left|\mathscr{H}_{\mathrm{a}}\right|^{2} t^{2} \hat{a}_{1}\left(\hat{a}_{\mathrm{a}}^{+} \hat{a}_{\mathrm{a}}-\hat{a}_{\mathrm{p}}^{+} \hat{a}_{\mathrm{p}}\right)\right] \exp \left(-\mathrm{i} \omega_{1} t\right) \\
& \hat{a}_{\mathrm{S}}(t)=\left[\hat{a}_{\mathrm{S}}-\mathrm{i} \mathscr{H}_{\mathrm{s}} t \hat{a}_{1} \hat{a}_{\mathrm{p}}^{+}+\frac{1}{2}\left|\mathscr{H}_{\mathrm{S}}\right|^{2} t^{2} \hat{a}_{\mathrm{s}}\left(\hat{a}_{1}^{+} \hat{a}_{1}-\hat{a}_{\mathrm{p}}^{+} \hat{a}_{\mathrm{p}}\right)\right. \\
& \left.-\frac{1}{2} \mathscr{H}_{\mathrm{s}} \mathscr{H}_{\mathrm{a}}^{*} t^{2} \hat{a}_{\mathrm{p}}^{+2} \hat{a}_{\mathrm{a}}+\frac{1}{2} \mathscr{H}_{\mathrm{s}} \mathscr{H}_{\mathrm{a}} t^{2} \hat{a}_{1}^{2} \hat{a}_{\mathrm{a}}^{+}\right] \exp \left(-\mathrm{i} \omega_{\mathrm{s}} t\right) \\
& \hat{a}_{\mathrm{a}}(t)=\left[\hat{a}_{\mathrm{a}}-\mathrm{i} \mathscr{H}_{\mathrm{a}} t \hat{a}_{1} \hat{a}_{\mathrm{p}}-\frac{1}{2}\left|\mathscr{H}_{\mathrm{a}}\right|^{2} t^{2} \hat{a}_{\mathrm{a}}\left(\hat{a}_{l}^{+} \hat{a}_{1}+\hat{a}_{\mathrm{p}}^{+} \hat{a}_{\mathrm{p}}+1\right)\right. \\
& \left.-\frac{1}{2} \mathscr{H}_{\mathrm{s}}^{*} \mathscr{H}_{\mathrm{a}} t^{2} \hat{a}_{\mathrm{p}}^{2} \hat{a}_{\mathrm{S}}-\frac{1}{2} \mathscr{H}_{\mathrm{s}} \mathscr{H}_{\mathrm{a}} t^{2} \hat{a}_{1}^{2} \hat{a}_{\mathrm{s}}^{+}\right] \exp \left(-\mathrm{i} \omega_{\mathrm{a}} t\right) \\
& \hat{a}_{\mathrm{p}}(t)=\left[\hat{a}_{\mathrm{p}}-\mathrm{i} \mathscr{H}_{\mathrm{s}} t \hat{a}_{1} \hat{a}_{\mathrm{S}}^{+}-\mathrm{i} \mathcal{H}_{\mathrm{a}}^{*} t \hat{a}_{1}^{+} \hat{a}_{\mathrm{a}}-\frac{1}{2}\left|\mathscr{H}_{\mathrm{s}}\right|^{2} t^{2} \hat{a}_{\mathrm{p}}\left(\hat{a}_{\mathrm{s}}^{+} \hat{a}_{\mathrm{S}}-\hat{a}_{1}^{+} \hat{a}_{1}\right)\right. \\
& \left.-\frac{1}{2}\left|\mathscr{H}_{\mathrm{a}}\right|^{2} \mid t^{2} \hat{a}_{\mathrm{p}}\left(\hat{a}_{1}^{+} \hat{a}_{1}-\hat{a}_{\mathrm{a}}^{+} \hat{a}_{\mathrm{a}}\right)\right] \exp \left(-\mathrm{i} \omega_{\mathrm{p}} t\right)
\end{aligned}
$$

where $\hat{a}_{\mathrm{j}} \equiv \hat{a}_{\mathrm{j}}(0)$.

\section{Time variation of the quantum characteristic functions}

In order to describe the statistical properties of nonlinear interaction between photons and phonons in Raman scattering, we use joint normally ordered characteristic functions. The joint normally ordered characteristic function for two different modes $i$ and $j$ is defined by the relation (Mehta and Sudarshan 1965, Mehta 1967, Glauber 1966, Peřina 1972)

$$
\begin{aligned}
& C_{N}\left(\beta_{i}, \beta_{j}, t\right)_{(i \neq j)}=\operatorname{Tr}\left[\hat{\rho}(0) \exp \left(\beta_{i} \hat{a}_{i}^{+}(t)+\beta_{j} \hat{a}_{i}^{+}(t)\right) \exp \left(-\beta_{i}^{*} \hat{a}_{i}(t)-\beta_{i}^{*} \hat{a}_{i}(t)\right)\right] \\
&(i, j=1, \mathrm{~S}, \mathrm{a}, \mathrm{p})
\end{aligned}
$$

where

$\left.\hat{\rho}(0)=\int \Phi_{\mathrm{N}}\left(\xi_{1} \xi_{\mathrm{s}}, \xi_{\mathrm{a}}, \xi_{\mathrm{p}}, 0\right) \xi_{1}, \xi_{\mathrm{s}}, \xi_{\mathrm{a}}, \xi_{\mathrm{p}}\right\rangle\left\langle\xi_{1}, \xi_{\mathrm{s}}, \xi_{\mathrm{a}}, \xi_{\mathrm{p}}\right| \mathrm{d}^{2} \xi_{\mathrm{l}} \mathrm{d}^{2} \xi_{\mathrm{s}} \mathrm{d}^{2} \xi_{\mathrm{a}} \mathrm{d}^{2} \xi_{\mathrm{p}}$

is the total density operator of the system in the Glauber-Sudarshan representation at the initial time $t=0$. The $\xi_{j}=\left|\xi_{j}\right| \exp \left(\mathrm{i} \phi_{j}\right)$ are the eigenvalues of the photon and phonon annihilation operators, $\phi_{i}$ denoting the initial phases of the modes. The joint normally ordered characteristic function for two modes contains all statistical information about 
the interaction between them. With the joint characteristic function available, we can easily determine the correlation between the number of bosons by means of photonphoton covariance functions and photon-phonon covariance functions

$$
\begin{aligned}
\left\langle\Delta W_{i} \Delta W_{j}\right\rangle \equiv & \left\langle\hat{a}_{i}^{+}(t) \hat{a}_{i}(t) \hat{a}_{i}^{+}(t) \hat{a}_{j}(t)\right\rangle-\left\langle\hat{a}_{i}^{+}(t) \hat{a}_{i}(t)\right\rangle\left\langle\hat{a}_{i}^{+}(t) \hat{a}_{j}(t)\right\rangle \\
= & \left.\frac{\partial^{4} C_{\mathrm{N}}\left(\beta_{i}, \beta_{i}, t\right)}{\partial \beta_{i} \partial\left(-\beta_{i}^{*}\right) \partial \beta_{j} \partial\left(-\beta_{i}^{*}\right)}\right|_{\beta_{i}=\beta_{i}=0} \\
& -\left.\left.\frac{\partial^{2} C_{\mathrm{N}}\left(\beta_{i}, \beta_{i}=0, t\right)}{\partial \beta_{i} \partial\left(-\beta_{i}^{*}\right)}\right|_{\beta_{i}=0} \frac{\partial^{2} C_{\mathrm{N}}\left(\beta_{i}=0, \beta_{j}, t\right)}{\partial \beta_{i} \partial\left(-\beta_{i}^{*}\right)}\right|_{\beta_{i}=0} \quad(i, j=1, \mathrm{~S}, \mathrm{a}, \mathrm{p}) .
\end{aligned}
$$

The covariance functions (3.3) may be positive, negative or zero. In the first case we have a correlation, whereas in the second we have an anticorrelation between bosons in two different modes. In the third case the bosons are not correlated. Putting $\beta_{i}=0$ or $\beta_{j}=0$ in equation (3.1) we obtain the characteristic functions for individual modes. With the help of these functions, the average numbers of photons and phonons and variances of intensity and higher-order moments for individual modes can be calculated. The boson variance of intensity is given by

$$
\begin{aligned}
\left\langle\left(\Delta W_{i}\right)^{2}\right\rangle & \equiv\left\langle\hat{a}_{i}^{+2}(t) \hat{a}_{i}^{2}(t)\right\rangle-\left\langle\hat{a}_{i}^{+}(t) \hat{a}_{i}(t)\right\rangle^{2} \\
& =\left.\frac{\partial^{4} C_{\mathrm{N}}\left(\beta_{i}, t\right)}{\partial^{2} \beta_{i} \partial^{2}\left(-\beta_{i}^{*}\right)}\right|_{\beta_{i}=0}-\left(\left.\frac{\partial^{2} C_{\mathrm{N}}\left(\beta_{i}, t\right)}{\partial \beta_{i} \partial\left(-\beta_{i}^{*}\right)}\right|_{\beta_{i}=0}\right)^{2} \quad(i=1, \mathrm{~S}, \mathrm{a}, \mathrm{p})
\end{aligned}
$$

where

$$
\left\langle\hat{a}_{i}^{+}(t) \hat{a}_{i}(t)\right\rangle=\left.\frac{\partial^{2} C_{\mathrm{N}}\left(\beta_{i}, t\right)}{\partial \beta_{i} \partial\left(-\beta_{i}^{*}\right)}\right|_{\beta_{t}=0}
$$

is the average number of bosons. When the variance of intensity is negative, we have the so-called antibunching of bosons (Peřina 1972), i.e. anticorrelation between photons or phonons in individual modes. Positive variance of intensity is evidence of correlation between bosons (bunching of bosons). If the value of the variance of intensity is zero, the bosons in a mode will be uncorrelated. Anticorrelation between modes $i$ and $j$ is associated with the non-existence of a joint normally ordered quasi-distribution function $\Phi_{\mathrm{N}}\left(\xi_{i}^{\prime}, \xi_{i}^{\prime}, t\right)$, which is defined by the inverse Fourier transform of the joint characteristic function (3.1)

$$
\Phi_{\mathrm{N}}\left(\xi_{i}^{\prime}, \xi_{j}^{\prime}, t\right)=\left(1 / \pi^{2}\right) \int C_{\mathrm{N}}\left(\beta_{i}, \beta_{j}, t\right) \exp \left(-\beta_{i} \xi_{i}^{*}-\beta_{j} \xi_{i}^{*}+\beta_{i}^{*} \xi_{i}^{\prime}+\beta_{i}^{*} \beta_{j}^{\prime}\right) \mathrm{d}^{2} \beta_{i} \mathrm{~d}^{2} \beta_{j} .
$$

The non-existence of the function $\Phi_{\mathrm{N}}\left(\xi_{i}^{\prime}, \xi_{j}^{\prime}, t\right)$ does not exclude the existence of quasi-distribution functions $\Phi_{\mathrm{N}}\left(\xi_{i}^{\prime}, t\right)$ and $\Phi_{\mathrm{N}}\left(\xi_{j}^{\prime}, t\right)$ separately for the modes $i$ and $j$ unless antibunching of bosons occurs in these modes.

\section{Anticorrelation effect between laser and Stokes modes}

In this Section we consider anticorrelation between the laser and Stokes photons. The joint normally ordered characteristic function for the incident laser mode and Stokes mode can be written using equation (3.1) in the form

$$
C_{\mathrm{N}}\left(\beta_{1}, \beta_{\mathrm{S}}, t\right)=\operatorname{Tr}\left[\hat{\rho}(0) \exp \left(\beta_{1} \hat{a}_{1}^{+}(t)+\beta_{\mathrm{S}} \hat{a}_{\mathrm{S}}^{+}(t)\right) \exp \left(-\beta_{1}^{*} \hat{a}_{1}(t)-\beta_{\mathrm{S}}^{*} \hat{a}_{\mathrm{S}}(t)\right)\right] \text {. }
$$


We evaluate the characteristic function (4.1) in the case when the initial states of the laser, Stokes and anti-Stokes modes are coherent states but the phonon mode is chaotic, i.e. when the initial total density operator of the system has the form

$\hat{\rho}(0)=\left(1 / \pi n_{\mathrm{p}}\right) \int \exp \left(-\left|\xi_{\mathrm{p}}\right|^{2} / n_{\mathrm{p}}\right)\left|\xi_{\mathrm{l}}, \xi_{\mathrm{s}}, \xi_{\mathrm{a}}, \xi_{\mathrm{p}}\right\rangle\left\langle\xi_{\mathrm{l}}, \xi_{\mathrm{s}}, \xi_{\mathrm{a}}, \xi_{\mathrm{p}}\right| \mathrm{d}^{2} \xi_{\mathrm{p}}$

The average number of phonons at the time $t=0$ is denoted by $n_{\mathrm{p}}$. Inserting the solutions (2.11) and (2.12) of the Heisenberg equation of motion into equation (4.1), performing normal ordering with the help of the Baker-Hausdorff identity up to $t^{2}$ and taking the trace, we obtain

$$
\begin{aligned}
C_{\mathrm{N}}\left(\beta_{1}, \beta_{\mathrm{S}}, t\right)= & \exp \left[\beta_{1} \xi_{1}^{*}(t)+\beta_{\mathrm{S}} \xi_{\mathrm{S}}^{*}(t)-\beta_{1}^{*} \xi_{1}(t)-\beta_{\mathrm{S}}^{*} \xi_{\mathrm{S}}(t)-\left|\beta_{1}\right|^{2} B_{1}(t)\right. \\
& \left.-\left|\beta_{\mathrm{S}}\right|^{2} B_{\mathrm{S}}(t)+\left(\frac{1}{2} \beta_{1}^{* 2} C_{1}(t)+\beta_{1}^{*} \beta_{\mathrm{S}}^{*} D_{\mathrm{IS}}(t)+\beta_{1} \beta_{\mathrm{S}}^{*} \bar{D}_{1 \mathrm{~S}}(t)+\mathrm{CC}\right)\right],
\end{aligned}
$$

where

$$
\begin{aligned}
& \xi_{1}(t)=\left[\xi_{1}-\frac{1}{2}\left|\mathscr{H}_{\mathrm{s}}\right|^{2} t^{2} \xi_{1}\left(\left|\xi_{\mathrm{s}}\right|^{2}+n_{\mathrm{p}}+1\right)+\frac{1}{2}\left|\mathscr{H}_{\mathrm{a}}\right|^{2} t^{2} \xi_{1}\left(\left|\xi_{\mathrm{a}}\right|^{2}-n_{\mathrm{p}}\right)\right] \exp \left(-\mathrm{i} \omega_{1} t\right) \\
& \xi_{\mathrm{s}}(t)=\left[\xi_{\mathrm{s}}+\frac{1}{2}\left|\mathscr{H}_{\mathbf{S}}\right|^{2} t^{2} \xi_{\mathrm{s}}\left(\left|\xi_{1}\right|^{2}-n_{\mathrm{p}}\right)+\frac{1}{2} \mathscr{H}_{\mathrm{S}} \mathscr{H}_{\mathrm{a}} t^{2} \xi_{1}^{2} \xi_{\mathrm{a}}^{*}\right] \exp \left(-\mathrm{i} \omega_{\mathrm{S}} t\right) \\
& C_{1}(t)=-\mathscr{H}_{\mathrm{s}}^{*} \mathscr{H}_{\mathrm{a}}^{*} t^{2} \xi_{\mathrm{s}} \xi_{\mathrm{a}}\left(2 n_{\mathrm{p}}+1\right) \exp \left(-2 \mathrm{i} \omega_{1} t\right) \\
& B_{1}(t)=\left|\mathscr{H}_{\mathrm{s}}\right|^{2} t^{2}\left|\xi_{\mathrm{s}}\right|^{2} n_{\mathrm{p}}+\left|\mathscr{H}_{\mathrm{a}}\right|^{2} t^{2}\left|\xi_{\mathrm{a}}\right|^{2}\left(n_{\mathrm{p}}+1\right) \\
& B_{\mathrm{S}}(t)=\left|\mathscr{H}_{\mathrm{S}}\right|^{2} t^{2}\left|\xi_{1}\right|^{2}\left(n_{\mathrm{p}}+1\right) \\
& D_{\mathrm{IS}}(\mathrm{t})=-\left|\mathscr{H}_{\mathrm{s}}\right|^{2} t^{2} \xi_{1} \xi_{\mathrm{s}}\left(n_{\mathrm{p}}+\frac{1}{2}\right) \exp \left[-\mathrm{i}\left(\omega_{1}+\omega_{\mathrm{s}}\right) t\right] \\
& \bar{D}_{\mathrm{IS}}(t)=-\mathscr{H}_{\mathrm{s}} \mathscr{H}_{\mathrm{a}} t^{2} \xi_{1} \xi_{\mathrm{a}}^{*}\left(n_{\mathrm{p}}+1\right) \exp \left[\mathrm{i}\left(\omega_{1}-\omega_{\mathrm{s}}\right) t\right] \text {. }
\end{aligned}
$$

The above characteristic function contains all statistical information about interaction between the laser and Stokes modes in spontaneous and stimulated Raman scattering. If only the spontaneous Raman effect takes place, then $\xi_{\mathrm{s}}=\xi_{\mathrm{a}}=0$ (the Stokes and anti-Stokes modes are in vacuum state at the time $t=0$ ). With the help of equations (3.4) and (4.3) we find the variances of intensity for the laser and Stokes radiation. We have

$$
\begin{aligned}
\left\langle\left(\Delta W_{1}\right)^{2}\right\rangle= & B_{1}^{2}(t)+2 B_{1}(t)\left|\xi_{1}(t)\right|^{2}+\left(C_{\mathrm{l}}(t) \xi_{1}^{* 2}(t)+\mathrm{CC}\right) \\
= & 2\left|\mathscr{H}_{\mathrm{S}}\right|^{2} t^{2}\left|\xi_{1}\right|^{2}\left|\xi_{\mathrm{s}}\right|^{2} n_{\mathrm{p}}+\left.2\left|\mathscr{H}_{\mathrm{a}}\right|^{2} t^{2} \xi_{1}\right|^{2}\left|\xi_{\mathrm{a}}\right|^{2}\left(n_{\mathrm{p}}+1\right) \\
& -t^{2}\left(2 n_{\mathrm{p}}+1\right)\left(\mathscr{H}_{\mathrm{S}} \mathscr{H}_{\mathrm{a}} \xi_{1}^{* 2} \xi_{\mathrm{s}} \xi_{\mathrm{a}}+\mathrm{CC}\right) \\
& \quad\left\langle\left(\Delta W_{\mathrm{s}}\right)^{2}\right\rangle=B_{\mathrm{S}}^{2}(t)+2 B_{\mathrm{S}}(t)\left|\xi_{\mathrm{s}}(t)\right|^{2}=2\left|\mathscr{H}_{\mathrm{s}}\right|^{2} t^{2}\left|\xi_{1}\right|^{2}\left|\xi_{\mathrm{s}}\right|^{2}\left(n_{\mathrm{p}}+1\right) .
\end{aligned}
$$

Similarly, using equations (3.3) and (4.3), we find the covariance function for the laser and Stokes photons:

$$
\begin{aligned}
\left\langle\Delta W_{1} \Delta W_{\mathrm{S}}\right\rangle= & \left|D_{\mathrm{IS}}(t)\right|^{2}+\left|\bar{D}_{\mathrm{IS}}(t)\right|^{2}+\left(D_{\mathrm{IS}}(t) \xi_{1}^{*}(t) \xi_{\mathrm{S}}^{*}(t)-\bar{D}_{1 \mathrm{~S}}(t) \xi_{1}(t) \xi_{\mathrm{S}}^{*}(t)+\mathrm{CC}\right) \\
& =-\left|\mathscr{H}_{\mathrm{s}}\right|^{2} t^{2}\left|\xi_{1}\right|^{2}\left|\xi_{\mathrm{s}}\right|^{2}\left(2 n_{\mathrm{p}}+1\right)+t^{2}\left(n_{\mathrm{p}}+1\right)\left(\mathscr{H}_{\mathrm{s}} \mathscr{H}_{\mathrm{a}} \xi_{1}^{* 2} \xi_{\mathrm{s}} \xi_{\mathrm{a}}+\mathrm{CC}\right) .
\end{aligned}
$$

In spontaneous Raman scattering, the variances of intensity (4.11), (4.12) and covariance function (4.13) are zero up to $t^{2}$. In the stimulated Raman effect, there occurs bunching of laser and Stokes photons. From equation (4.13) we see that the covariance function for the laser and Stokes photons depends on the phases $\phi_{\mathrm{l}}, \phi_{\mathrm{S}}$ and $\phi_{\mathrm{a}}$ of the initial laser, Stokes and anti-Stokes modes respectively. On assuming the phase 
matching

$$
\Delta \boldsymbol{k}_{\mathrm{S}, \mathrm{a}} \equiv \boldsymbol{k}_{\mathrm{l}} \mp \boldsymbol{k}_{\mathrm{p}}-\boldsymbol{k}_{\mathrm{S}, \mathrm{a}}=0
$$

and the following relation for the phases $\Delta \phi \equiv 2 \phi_{1}-\phi_{\mathrm{S}}-\phi_{\mathrm{a}}=\pi$, we obtain the maximal anticorrelation between the laser and Stokes photons

$\left.\left\langle\Delta W_{1} \Delta W_{\mathrm{s}}\right\rangle=-\mathscr{H}_{\mathrm{s}}^{2} t^{2}\left|\xi_{1}\right|^{2}\left|\xi_{\mathrm{s}}\right|^{2}\left(2 n_{\mathrm{p}}+1\right)-2 \mathscr{H}_{\mathrm{s}} \mathscr{H}_{\mathrm{a}} t^{2}\left|\xi_{1}\right|^{2}\left|\xi_{\mathrm{s}} \| \xi_{\mathrm{a}}\right| n_{\mathrm{p}}+1\right)$.

For $\Delta k_{\mathrm{S}, \mathrm{a}}=0$ and $\Delta \phi=\pi / 2$, we have

$$
\left\langle\Delta W_{1} \Delta W_{\mathrm{s}}\right\rangle=-\mathscr{H}_{\mathrm{s}}^{2} t^{2}\left|\xi_{\mathrm{l}}\right|^{2}\left|\xi_{\mathrm{s}}\right|^{2}\left(2 n_{\mathrm{p}}+1\right)
$$

From equation (4.13) we see that anticorrelation between the laser and Stokes photons does not depend on the phases $\phi_{1}, \phi_{\mathrm{S}}$ and $\phi_{\mathrm{a}}$ when anti-Stokes radiation is in the vacuum state at the time $t=0$ (only stimulated Stokes emission takes place). One easily notes that, even at $n_{\mathrm{p}}=0$, bunching of laser and Stokes photons and anticorrelation between them exist as a result of phonon vacuum fluctuations:

$$
\begin{aligned}
& \left\langle\left(\Delta W_{1}\right)^{2}\right\rangle=2\left|\mathscr{H}_{\mathrm{a}}\right|^{2} t^{2}\left|\xi_{1}\right|^{2}\left|\xi_{\mathrm{S}}\right|^{2}-t^{2}\left(\mathscr{H}_{\mathrm{S}} \mathscr{H}_{\mathrm{a}} \xi_{1}^{* 2} \xi_{\mathrm{s}} \xi_{\mathrm{a}}+\mathrm{CC}\right) \\
& \left\langle\left(\Delta W_{\mathrm{S}}\right)^{2}\right\rangle=2\left|\mathscr{H}_{\mathrm{S}}\right|^{2} t^{2}\left|\xi_{1}\right|^{2}\left|\xi_{\mathrm{S}}\right|^{2} \\
& \left\langle\Delta W_{\mathrm{l}} \Delta W_{\mathrm{S}}\right\rangle=-\left|\mathscr{H}_{\mathrm{S}}\right|^{2} t^{2}\left|\xi_{\mathrm{l}}\right|^{2}\left|\xi_{\mathrm{S}}\right|^{2}+t^{2}\left(\mathscr{H}_{\mathrm{S}} \mathscr{H}_{\mathrm{a}} \xi_{1}^{* 2} \xi_{\mathrm{s}} \xi_{\mathrm{a}}+\mathrm{CC}\right) .
\end{aligned}
$$

One can prove that, when the initial state of the system is a coherent state in all four modes the covariance function for the laser and Stokes modes is equivalent to the above function (coherent phonon mode describing acoustical wave Brillouin scattering). Therefore the magnitude of the anticorrelation between the laser and Stokes photons depends on the initial statistics of the phonons. When anticorrelation between the laser and Stokes photons occurs the joint normally ordered quasi-distribution function $\Phi_{\mathrm{N}}\left(\xi_{1}^{\prime}, \xi_{\mathrm{S}}^{\prime}, t\right)$ for the laser and Stokes modes does not exist, but quasi-distribution functions $\Phi_{\mathrm{N}}\left(\xi_{1}^{\prime}, t\right)$ and $\Phi_{\mathrm{N}}\left(\xi_{S}^{\prime}, t\right)$ exist separately for the laser and Stokes modes. Let us examine these functions. Putting $\beta_{1}=0$ in equation (4.3) we obtain

$$
C_{\mathrm{N}}\left(\beta_{\mathrm{S}}, t\right)=\exp \left(\beta_{\mathrm{S}} \xi_{\mathrm{S}}^{*}(t)-\beta_{\mathrm{S}}^{*} \xi_{\mathrm{S}}(t)-\left|\beta_{\mathrm{S}}\right|^{2} B_{\mathrm{S}}(t)\right)
$$

The quasi-distribution function for the Stokes mode obtained by using the inverse Fourier transform of the function (4.19) is given by a Gaussian function:

$$
\Phi_{\mathrm{N}}\left(\xi_{\mathrm{S}}^{\prime}, t\right)=\left(1 / \pi B_{\mathrm{S}}(t)\right) \exp \left(-\left|\xi_{\mathrm{S}}^{\prime}-\xi_{\mathrm{S}}(t)\right|^{2} / B_{\mathrm{S}}(t)\right)
$$

where the variance $B_{\mathrm{S}}(t)=\left|\mathscr{H}_{\mathrm{S}}\right|^{2} t^{2}\left|\xi_{!}\right|^{2}\left(n_{\mathrm{p}}+1\right)$ describes the noise present in the Stokes field from spontaneous Raman scattering. Therefore, the above quasi-distribution exhibits a superposition of noise on the coherent amplification of the stimulated Raman effect. Similarly, we obtain the quasi-distribution function for the laser field neglecting for simplicity the rotational terms $C_{\mathrm{l}}(t)$ and $C_{1}^{*}(t)$

$$
\Phi_{\mathrm{N}}\left(\xi_{1}^{\prime}, t\right)=\left(1 / \pi B_{1}(t)\right) \exp \left(-\left|\xi_{1}^{\prime}-\xi_{1}(t)\right|^{2} / B_{1}(t)\right)
$$

where the variance $B_{\mathrm{l}}(t)$ is given by equation (4.7).

Therefore, the initially coherent laser radiation does not remain coherent for $t>0$. As seen from equations (4.20) and (4.21), these are functions well known from nonlinear optics (Walls 1973, Agrawal and Mehta 1974, Peřina 1972, Peŕinová and Peřina 1978). The joint normally ordered characteristic function for spontaneous Raman scattering $\left(\xi_{\mathrm{s}}=\xi_{\mathrm{a}}=0\right)$ has the form:

$$
C_{\mathrm{N}}\left(\beta_{1}, \beta_{\mathrm{S}}, t\right)=\exp \left(\beta_{1} \xi_{1}^{*}(t)-\beta_{1}^{*} \xi_{1}(t)-\left|B_{\mathrm{S}}\right|^{2} B_{\mathrm{S}}(t)\right)
$$


where

$$
\begin{aligned}
& \xi_{1}(t)=\left[\xi_{1}-\frac{1}{2}\left|\mathscr{H}_{\mathrm{S}}\right|^{2} t^{2} \xi_{1}\left(n_{\mathrm{p}}+1\right)-\frac{1}{2}\left|\mathscr{H}_{\mathrm{a}}\right|^{2} t^{2} \xi_{1} n_{\mathrm{p}}\right] \exp \left(-\mathrm{i} \omega_{1} t\right) \\
& B_{\mathrm{S}}(t)=\left|\mathscr{H}_{\mathrm{S}}\right|^{2} t^{2}\left|\xi_{1}\right|^{2}\left(n_{\mathrm{p}}+1\right) .
\end{aligned}
$$

Taking the inverse Fourier transform (3.6) of the function (4.22), we obtain

$$
\Phi_{\mathrm{N}}\left(\xi_{1}^{\prime}, \xi_{\mathrm{S}}^{\prime} t\right)=\Phi_{\mathrm{N}}\left(\xi_{1}^{\prime}, t\right) \Phi_{\mathrm{N}}\left(\xi_{\mathrm{s}}^{\prime}, t\right)
$$

where

$$
\begin{aligned}
& \Phi_{\mathrm{N}}\left(\xi_{1}^{\prime}, t\right)=\sigma^{(2)}\left(\xi_{1}^{\prime}-\xi_{1}(t)\right) \\
& \Phi_{\mathrm{N}}\left(\xi_{\mathrm{S}}^{\prime}, t\right)=\left(1 / \pi B_{\mathrm{S}}(t)\right) \exp \left(-\left|\xi_{\mathrm{S}}^{\prime}\right|^{2} / B_{\mathrm{S}}(t)\right) .
\end{aligned}
$$

It may be noted that in spontaneous Raman scattering the laser and Stokes modes are uncoupled up to $t^{2}$. The quasi-distribution function for the laser field (4.26) is given by a two-dimensional Dirac delta function, meaning that the initially coherent laser mode remains coherent for $t>0$. The Stokes mode is described by the Gaussian function (4.27) centred at zero with the variance $B_{\mathrm{S}}(t)=\left|\mathscr{H}_{\mathrm{S}}\right|^{2} t^{2}\left|\xi_{1}\right|^{2}\left(n_{\mathrm{p}}+1\right)$ describing noise from spontaneous Raman scattering.

In order to study how anticorrelation between the laser and Stokes photons depends on the statistical properties of the initial Stokes and anti-Stokes states, we evaluate the characteristic function (4.1) when the laser field is coherent but the Stokes, anti-Stokes and phonon modes are chaotic before the interaction, i.e. when

$$
\begin{aligned}
\hat{\rho}(0)=\frac{1}{\pi^{3} n_{\mathrm{S}} n_{\mathrm{a}} n_{\mathrm{p}}} \int \exp \left(-\frac{\left|\xi_{\mathrm{s}}\right|^{2}}{n_{\mathrm{S}}}-\frac{\left|\xi_{\mathrm{a}}\right|^{2}}{n_{\mathrm{a}}}-\frac{\left|\xi_{\mathrm{p}}\right|^{2}}{n_{\mathrm{p}}}\right) \\
\times\left|\xi_{\mathrm{l}}, \xi_{\mathrm{s}}, \xi_{\mathrm{a}}, \xi_{\mathrm{p}}\right\rangle\left\langle\xi_{\mathrm{l}}, \xi_{\mathrm{s}}, \xi_{\mathrm{a}}, \xi_{\mathrm{p}}\right| \mathrm{d}^{2} \xi_{\mathrm{S}} \mathrm{d}^{2} \xi_{\mathrm{a}} \mathrm{d}^{2} \xi_{\mathrm{p}}
\end{aligned}
$$

where $n_{\mathrm{S}}$ and $n_{\mathrm{a}}$ are the average numbers of the Stokes and anti-Stokes photons respectively at the time $t=0$. Using the.same procedure as in the first case, we obtain

$$
\begin{aligned}
C_{\mathrm{N}}\left(\beta_{1}, \beta_{\mathrm{S}}, t\right)= & \exp \left(\beta_{1} \xi_{1}^{*}(t)-\beta_{1}^{*} \xi_{1}(t)-\left|\beta_{1}\right|^{2} B_{1}(t)-\left|\beta_{\mathrm{S}}\right|^{2} B_{\mathrm{S}}(t)\right. \\
& \left.-\beta_{\mathrm{I}}\left|\beta_{\mathrm{S}}\right|^{2} G_{\mathrm{ISS}}^{*}(t)+\beta_{\mathrm{I}}^{*}\left|\beta_{\mathrm{S}}\right|^{2}{ }^{2} G_{\mathrm{ISS}}(t)+\left|\beta_{1}\right|^{2}\left|\beta_{\mathrm{S}}\right|^{2} B_{\mathrm{IS}}(t)\right)
\end{aligned}
$$

where

$$
\begin{aligned}
\xi_{1}(t)=\left[\xi_{1}-\frac{1}{2}\left|\mathscr{H}_{\mathrm{S}}\right|^{2} t^{2} \xi_{1}\left(n_{\mathrm{S}}+n_{\mathrm{p}}+1\right)+\frac{1}{2}\left|\mathscr{H}_{\mathrm{a}}\right|^{2} t^{2} \xi_{1}\left(n_{\mathrm{a}}-n_{\mathrm{p}}\right)\right] \exp \left(-\mathrm{i} \omega_{1} t\right) \\
B_{1}(t)=\left|\mathscr{H}_{\mathrm{S}}\right|^{2} t^{2} n_{\mathrm{S}} n_{\mathrm{p}}+\left|\mathscr{H}_{\mathrm{a}}\right|^{2} t^{2} n_{\mathrm{a}}\left(n_{\mathrm{p}}+1\right) \\
B_{\mathrm{S}}(t)=\left\langle\hat{a}_{\mathrm{S}}^{+}(t) \hat{a}_{\mathrm{S}}(t)\right\rangle=n_{\mathrm{S}}+\left|\mathscr{H}_{\mathrm{S}}\right|^{2} t^{2}\left|\xi_{1}\right|^{2}\left(n_{\mathrm{S}}+n_{\mathrm{p}}+1\right)-\left|\mathscr{H}_{\mathrm{S}}\right|^{2} t^{2} n_{\mathrm{S}} n_{\mathrm{p}} \\
G_{\mathrm{ISS}}(t)=-\left|\mathscr{H}_{\mathrm{S}}\right|^{2} t^{2} \xi_{1} n_{\mathrm{S}}\left(n_{\mathrm{p}}+\frac{1}{2} n_{\mathrm{S}}+\frac{1}{2}\right) \exp \left(-\mathrm{i} \omega_{\mathrm{l}} t\right) \\
B_{1, \mathrm{~S}}(t)=\left|\mathscr{H}_{\mathrm{S}}\right|^{2} t^{2} n_{\mathrm{S}}^{2} n_{\mathrm{p}} .
\end{aligned}
$$

The variances of intensity and covariance function have the form

$$
\begin{aligned}
\left\langle\left(\Delta W_{1}\right)^{2}\right\rangle= & B_{1}^{2}(t)+2 B_{1}(t)\left|\xi_{1}(t)\right|^{2} \\
& =2\left|\mathscr{L}_{\mathrm{S}}\right|^{2} t^{2}\left|\xi_{1}\right|^{2} n_{\mathrm{S}} n_{\mathrm{p}}+2\left|\mathscr{H}_{\mathrm{a}}\right|^{2} t^{2}\left|\xi_{1}\right|^{2} n_{\mathrm{a}}\left(n_{\mathrm{p}}+1\right) \\
\left\langle\left(\Delta W_{\mathrm{S}}\right)^{2}\right\rangle= & B_{\mathrm{S}}^{2}(t)=n_{\mathrm{S}}^{2}+2\left|\mathscr{H}_{\mathrm{S}}\right|^{2} t^{2}\left|\xi_{\mathrm{S}}\right|^{2} n_{\mathrm{S}}\left(n_{\mathrm{S}}+n_{\mathrm{p}}+1\right)-2\left|\mathscr{H}_{\mathrm{S}}\right|^{2} t^{2} n_{\mathrm{S}}^{2} n_{\mathrm{p}} \\
\left\langle\Delta W_{1} \Delta W_{\mathrm{S}}\right\rangle & =G_{\mathrm{ISS}}(t) \xi_{1}^{*}(t)+G_{1 \mathrm{SS}}^{*}(t) \xi_{1}(t)+B_{\mathrm{IS}}(t) \\
& \left.=-\left|\mathscr{H}_{\mathrm{S}}\right|^{2} t^{2}\left|\xi_{1}\right|^{2} n_{\mathrm{S}}\left(2 n_{\mathrm{p}}+1\right)-\left.\left|\mathscr{H}_{\mathrm{S}}\right|^{2} t^{2} n_{\mathrm{S}}^{2}|| \xi_{\mathrm{S}}\right|^{2}-n_{\mathrm{p}}\right) .
\end{aligned}
$$


The above expressions lead to the following results: (i) the value of the covariance function (4.37) is negative (in practice, the average number of the laser photons is more than the average number of the phonons at the time $t=0$, i.e. $\left|\xi_{1}\right|^{2}>n_{\mathrm{p}}$ (Wang 1969)); (ii) the bunching of Stokes photons is higher for an initially chaotic than for a coherent Stokes field (relations (4.12) and (4.36) respectively); (iii) the bunching of laser photons and the anticorrelation between the laser and Stokes photons do not depend on the initial phases of the photon modes; (iv) the anticorrelation effect (4.37) and the bunching of photons (4.35) and (4.36) take place even at $n_{\mathrm{p}}=0$, but do not exist in the absence of stimulated Raman scattering; $(v)$ no joint normally ordered quasi-distribution function $\Phi_{\mathrm{N}}\left(\xi_{1}^{\prime}, \xi_{\mathrm{s}}^{\prime}, t\right)$ exists for the laser and Stokes modes in the presence of a stimulated Raman effect; (vi) normally ordered quasi-distribution functions $\Phi_{\mathrm{N}}\left(\xi_{\mathrm{i}}^{\prime}, t\right)$ and $\Phi_{\mathrm{N}}\left(\xi_{\mathrm{S}}^{\prime}, t\right)$ exist separately for the laser and Stokes modes in the presence of stimulated Raman scattering and are given by equation (4.21) with $\left|\xi_{\mathrm{s}}\right|^{2}=n_{\mathrm{S}}$ and $\left|\xi_{\mathrm{a}}\right|^{2}=n_{\mathrm{a}}$ and

$$
\Phi_{\mathrm{N}}\left(\xi_{\mathrm{S}}^{\prime}, t\right)=\left(1 / \pi B_{\mathrm{S}}(t)\right) \exp \left(-\left|\xi_{\mathrm{S}}^{\prime}\right|^{2} / B_{\mathrm{S}}(t)\right)
$$

respectively. The variance $B_{\mathrm{S}}(t)$ of the Gaussian function (4.38) is given by the average number of Stokes photons (4.32) and describes the degree of chaos in the Stokes mode from spontaneous and stimulated Raman scattering. Therefore, the initially chaotic Stokes mode remains chaotic for $t>0$.

\section{Anticorrelation effect between the laser and anti-Stokes modes}

In this Section we consider the existence problem of anticorrelation between laser and anti-Stokes photons. When the initial total density operator is given by equation (4.2), the joint normally ordered characteristic function for the laser and anti-Stokes fields is of the form:

$$
\begin{aligned}
C_{\mathrm{N}}\left(\beta_{1}, \beta_{\mathrm{a}}, t\right)= & \exp \left[\beta_{1} \xi_{1}^{*}(t)+\beta_{\mathrm{a}} \xi_{\mathrm{a}}^{*}(t)-\beta_{1}^{*} \xi_{1}(t)-\beta_{\mathrm{a}}^{*} \xi_{\mathrm{a}}(t)\right. \\
& -\left|\beta_{1}\right|^{2} B_{1}(t)-\left|\beta_{\mathbf{a}}\right|^{2} B_{\mathbf{a}}(t) \\
& \left.+\left(\frac{1}{2} \beta_{1}^{* 2}(t) C_{1}(t)+\beta_{1}^{*} \beta_{\mathrm{a}}^{*} D_{\mathrm{la}}(t)+\beta_{1} \beta_{\mathrm{a}}^{*} \bar{D}_{\mathrm{la}}(t)+\mathrm{CC}\right)\right]
\end{aligned}
$$

where

$$
\begin{aligned}
\xi_{1}(t)=\left[\xi_{1}-\frac{1}{2}\left|\mathscr{H}_{\mathrm{S}}\right|^{2} t^{2} \xi_{1}\left(\left|\xi_{\mathrm{s}}\right|^{2}+n_{\mathrm{p}}+1\right)+\frac{1}{2}\left|\mathscr{H}_{\mathrm{a}}\right|^{2} t^{2} \xi_{1}\left(\left|\xi_{\mathrm{a}}\right|^{2}-n_{\mathrm{p}}\right)\right] \exp \left(-\mathrm{i} \omega_{1} t\right) \\
\xi_{\mathrm{a}}(t)=\left[\xi_{\mathrm{a}}-\frac{1}{2}\left[\left.\mathscr{H}_{\mathrm{a}}\right|^{2} t^{2} \xi_{\mathrm{a}}\left[\left|\xi_{1}\right|^{2}+n_{\mathrm{p}}+1\right]-\frac{1}{2} \mathscr{H}_{\mathrm{s}} \mathscr{H}_{\mathrm{a}} t^{2} \xi_{1}^{2} \xi_{\mathrm{a}}^{*}\right] \exp \left(-\mathrm{i} \omega_{\mathrm{a}} t\right)\right. \\
C_{\mathrm{l}}(t)=-\mathscr{H}_{\mathrm{S}}^{*} \mathscr{H}_{\mathrm{a}}^{*} t \xi_{\mathrm{s}} \xi_{\mathrm{a}}\left(2 n_{\mathrm{p}}+1\right) \exp \left(-2 \mathrm{i} \omega_{1} t\right) \\
B_{1}(t)=\left|\mathscr{H}_{\mathrm{S}}\right|^{2} t^{2}\left|\xi_{\mathrm{s}}\right|^{2} n_{\mathrm{p}}+\left|\mathscr{H}_{\mathrm{a}}\right|^{2} t^{2}\left|\xi_{\mathrm{a}}\right|^{2}\left(n_{\mathrm{p}}+1\right) \\
B_{\mathrm{a}}(t)=\left|\mathscr{H}_{\mathrm{a}}\right|^{2} t^{2}\left|\xi_{1}\right|^{2} n_{\mathrm{p}} \\
D_{\mathrm{la}}(t)=-\left|\mathscr{H}_{\mathrm{a}}\right|^{2} t^{2} \xi_{1} \xi_{\mathrm{a}}\left(n_{\mathrm{p}}+\frac{1}{2}\right) \exp \left[-\mathrm{i}\left(\omega_{1}+\omega_{\mathrm{a}}\right) t\right] \\
\bar{D}_{\mathrm{la}}(t)=-\mathscr{H}_{\mathrm{S}} \mathscr{H}_{\mathrm{a}} t^{2} \xi_{1} \xi_{\mathrm{s}}^{*} n_{\mathrm{p}} \exp \left[\mathrm{i}\left(\omega_{1}-\omega_{\mathrm{a}}\right) t\right] .
\end{aligned}
$$

The variance of intensity for the laser field is given by equation (4.11). From equations (5.1) and (3.4) we find

$$
\left\langle\left(\Delta W_{\mathrm{a}}\right)^{2}\right\rangle=B_{\mathrm{a}}^{2}(t)+2 B_{\mathrm{a}}(t)\left|\xi_{\mathrm{a}}(t)\right|^{2}=2\left|\mathscr{H}_{\mathrm{a}}\right|^{2} t^{2}\left|\xi_{\mathrm{l}}\right|^{2}\left|\xi_{\mathrm{a}}\right|^{2} n_{\mathrm{p}}
$$


The above relation shows that bunching of anti-Stokes photons occurs in stimulated Raman scattering. The covariance function for the laser and anti-Stokes field is given by

$$
\begin{aligned}
\left\langle\Delta W_{1} \Delta W_{\mathrm{a}}\right\rangle & =\left|D_{\mathrm{la}}(t)\right|^{2}+\left|\bar{D}_{\mathrm{la}}(t)\right|^{2}+\left(D_{\mathrm{la}}(t) \xi_{1}^{*}(t) \xi_{\mathrm{a}}^{*}(t)-\bar{D}_{\mathrm{la}}(t) \xi_{\mathrm{la}}(t) \xi_{1}(\mathrm{t}) \xi_{\mathrm{a}}^{*}(t)+\mathrm{CC}\right) \\
& =-\left|\mathscr{H}_{\mathrm{a}}\right|^{2} t^{2}\left|\xi_{1}\right|^{2}\left|\xi_{\mathrm{a}}\right|^{2}\left(2 n_{\mathrm{p}}+1\right)+t^{2} n_{\mathrm{p}}\left(\mathscr{H}_{\mathrm{s}} \mathscr{H}_{\mathrm{a}} \xi_{1}^{* 2} \xi_{\mathrm{S}} \xi_{\mathrm{a}}+\mathrm{CC}\right) .
\end{aligned}
$$

Assuming phase matching $\Delta \boldsymbol{k}_{\mathrm{S}, \mathrm{a}}=0$ and putting $\Delta \phi=\pi$ we obtain the maximal anticorrelation between the laser and anti-Stokes photons

$\left\langle\Delta W_{1} \Delta W_{\mathrm{a}}\right\rangle=-\mathscr{H}_{\mathrm{a}}^{2} t^{2}\left|\xi_{1}\right|^{2}\left|\xi_{\mathrm{a}}\right|^{2}\left(2 n_{\mathrm{p}}+1\right)-2 \mathscr{H}_{\mathrm{s}} \mathscr{H}_{\mathrm{a}} t^{2}\left|\xi_{1}\right|^{2}\left|\xi_{\mathrm{s}}\right|\left|\xi_{\mathrm{a}}\right| n_{\mathrm{p}}$

For $\Delta \boldsymbol{k}_{\mathrm{S}, \mathrm{a}}=0$ and $\Delta \phi=\pi / 2$ we have

$$
\left\langle\Delta W_{1} \Delta W_{\mathrm{a}}\right\rangle=-\mathscr{H}_{\mathrm{a}}^{2} t^{2}\left|\xi_{1}\right|^{2}\left|\xi_{\mathrm{a}}\right|^{2}\left(2 n_{\mathrm{p}}+1\right) .
$$

One may note that the phase-dependent terms in equation (5.10) equal zero when stimulated anti-Stokes radiation takes place only. If initially $n_{p}=0$, the relations $(5.9)$ and (5.10) become

$$
\begin{aligned}
& \left\langle\left(\Delta W_{\mathrm{a}}\right)^{2}=0\right. \\
& \left\langle\Delta W_{1} \Delta W_{\mathrm{a}}\right\rangle=-\left|\mathscr{H}_{\mathrm{a}}\right|^{2} t^{2}\left|\xi_{1}\right|^{2}\left|\xi_{\mathrm{a}}\right|^{2} .
\end{aligned}
$$

It can be proved that the covariance function (5.14) is equivalent to the covariance function for the laser and anti-Stokes fields when all modes are in coherent states at the time $t=0$. From equation (5.13) we see that the anti-Stokes field tends to be coherent during the interaction. No identical effect occurs for the Stokes mode (see equation (4.12)).

The existence of anticorrelation between the laser and anti-Stokes photons excludes the existence of a joint normally ordered quasi-distribution function $\Phi_{\mathrm{N}}\left(\xi_{1}^{\prime}, \xi_{\mathrm{a}}^{\prime}, t\right)$ for the laser and anti-Stokes modes. The quasi-distribution function for the laser mode is given by equation (4.21), whereas that for the anti-Stokes mode is given by

$$
\Phi_{\mathrm{N}}\left(\xi_{\mathrm{a}}^{\prime}, t\right)=\left(1 / \pi B_{\mathrm{a}}(t)\right) \exp \left(-\left|\xi_{\mathrm{a}}^{\prime}-\xi_{\mathrm{a}}(t)\right|^{2} / B_{\mathrm{a}}(t)\right) .
$$

This is a Gaussian function centred at $\xi_{\mathrm{a}}(t)$ with variance given by (5.6). If $n_{\mathrm{p}}=0$, the function (5.15) tends to the Dirac delta function

$$
\Phi_{\mathrm{N}}\left(\xi_{\mathrm{a}}^{\prime}, t\right)=\delta^{(2)}\left(\xi_{\mathrm{a}}^{\prime}-\xi_{\mathrm{a}}(t)\right) \text {. }
$$

Therefore, when $n_{\mathrm{p}}=0$ the anti-Stokes mode remains coherent at $t>0$. In spontaneous Raman scattering the laser and anti-Stokes modes, likewise to the laser and Stokes modes, are uncoupled; therefore

$$
\Phi_{\mathrm{N}}\left(\xi_{1}^{\prime}, \xi_{\mathrm{a}}^{\prime} t\right)=\Phi_{\mathrm{N}}\left(\xi_{1}^{\prime}, t\right) \Phi_{\mathrm{N}}\left(\xi_{\mathrm{a}}^{\prime}, t\right)
$$

where $\Phi_{\mathrm{N}}\left(\xi_{1}^{\prime}, t\right)$ and $\Phi_{\mathrm{N}}\left(\xi_{\mathrm{a}}^{\prime}, t\right)$ are given by equation (4.26) and

$$
\Phi_{\mathrm{N}}\left(\xi_{\mathrm{a}}^{\prime}, t\right)=\left(1 / \pi B_{\mathrm{a}}(t)\right) \exp \left(-\left|\xi_{\mathrm{a}}^{\prime}\right|^{2} / B_{\mathrm{a}}(t)\right)
$$

respectively. The variance of the function (5.18) is given by (5.6) and describes the noise from spontaneous Raman scattering. We see from equation (5.18) that the anti-Stokes mode is in the vacuum state when $n_{\mathrm{p}}=0$.

If, in the initial state, the laser mode is coherent but the phonon, Stokes and anti-Stokes modes are chaotic, i.e. when the total density operator at the time $t=0$ is given by equation (4.28), the joint normally ordered characteristic function for the laser 
and anti-Stokes field is given by

$$
\begin{aligned}
C_{\mathrm{N}}\left(\beta_{1}, \beta_{\mathrm{a}}, t\right)= & \exp \left(\beta_{1} \xi_{1}^{*}(t)-\beta_{1}^{*} \xi_{1}(t)-\left|\beta_{1}\right|^{2} B_{1}(t)-\left|\beta_{\mathrm{a}}\right|^{2} B_{\mathrm{a}}(t)-\beta_{1}\left|\beta_{\mathrm{a}}\right|^{2} G_{\text {laa }}^{*}(t)\right. \\
& \left.+\beta_{1}^{*}\left|\beta_{\mathrm{a}}\right|^{2} G_{\mathrm{laa}}(t)+\left|\beta_{1}\right|^{2}\left|\beta_{\mathrm{a}}\right|^{2} B_{\mathrm{la}}(t)\right)
\end{aligned}
$$

where

$$
\begin{gathered}
\xi_{1}(t)=\left[\xi_{1}-\frac{1}{2}\left|\mathscr{H}_{\mathrm{s}}\right|^{2} t^{2} \xi_{1}\left(n_{\mathrm{S}}+n_{\mathrm{p}}+1\right)+\frac{1}{2}\left|\mathscr{H}_{\mathrm{a}}\right|^{2} t^{2} \xi_{\mathrm{l}}\left(n_{\mathrm{a}}-n_{\mathrm{p}}\right)\right] \exp \left(-\mathrm{i} \omega_{1} t\right) \\
B_{1}(t)=\left|\mathscr{H}_{\mathrm{S}}\right|^{2} t^{2} n_{\mathrm{s}} n_{\mathrm{p}}+\left|\mathscr{H}_{\mathrm{a}}\right|^{2} t^{2} n_{\mathrm{a}}\left(n_{\mathrm{p}}+1\right) \\
B_{\mathrm{a}}(t)=n_{\mathrm{a}}+\left|\mathscr{H}_{\mathrm{a}}\right|^{2} t^{2}\left|\xi_{1}\right|^{2}\left(n_{\mathrm{p}}-n_{\mathrm{a}}\right)-\left|\mathscr{H}_{\mathrm{a}}\right|^{2} t^{2} n_{\mathrm{a}}\left(n_{\mathrm{p}}+1\right) \\
G_{1 \mathrm{aa}}(t)=-\left|\mathscr{H}_{\mathrm{a}}\right|^{2} t^{2} \xi_{\mathrm{l}} n_{\mathrm{a}}\left(n_{\mathrm{p}}-\frac{1}{2} n_{\mathrm{a}}+\frac{1}{2}\right) \\
B_{1 \mathrm{a}}(t)=\left|\mathscr{H}_{\mathrm{a}}\right|^{2} t^{2} n_{\mathrm{a}}^{2}\left(n_{\mathrm{p}}+1\right) .
\end{gathered}
$$

The variance of intensity for the anti-Stokes radiation is determined by the following relation with accuracy up to $t^{2}$ :

$$
\left\langle\left(\Delta W_{\mathrm{a}}\right)^{2}\right\rangle=B_{\mathrm{a}}^{2}(t)=\left\langle\hat{a}_{\mathrm{a}}^{+}(t) \hat{a}_{\mathrm{a}}(t)\right\rangle^{2} .
$$

The covariance function $\left\langle\Delta W_{1} \Delta W_{\mathrm{a}}\right\rangle$ has the form

$$
\begin{aligned}
\left\langle\Delta W_{1} \Delta W_{\mathrm{a}}\right\rangle & =G_{\mathrm{laa}}(t) \xi_{1}^{*}(t)+G_{\mathrm{la}}^{*}(t) \xi_{1}(t)+B_{\mathrm{la}}(t) \\
& =-\left|\mathscr{H}_{\mathrm{a}}\right|^{2} t^{2}\left|\xi_{1}\right|^{2} n_{\mathrm{a}}\left(2 n_{\mathrm{p}}+1\right)+\left|\mathscr{H}_{\mathrm{a}}\right|^{2} t^{2} n_{a}^{2}\left(\left|\xi_{\mathrm{l}}\right|^{2}+n_{\mathrm{p}}+1\right) .
\end{aligned}
$$

The function (5.26) is negative when $\left|\xi_{1}\right|^{2}>n_{\mathrm{p}}>n_{\mathrm{a}}$ which, in practice, takes place. Therefore we have

$$
\left\langle\Delta W_{1} \Delta W_{\mathrm{a}}\right\rangle<0 \text {. }
$$

If $n_{\mathrm{p}}=0$ the function (5.26) is positive and we have the correlation effect

$$
\left\langle\Delta W_{\mathrm{l}} \Delta W_{\mathrm{a}}\right\rangle=\left|\mathscr{H}_{\mathrm{a}}\right|^{2} t^{2}\left[n_{\mathrm{a}}^{2}+n_{\mathrm{a}}\left|\xi_{1}\right|^{2}\left(n_{\mathrm{a}}-1\right)\right]>0 .
$$

One may note that for the laser and Stokes photons, if $n_{p}=0$ anticorrelation occurs. In the presence of the anticorrelation effect (5.27) the joint normally ordered quasidistribution function $\Phi_{\mathrm{N}}\left(\xi_{1}^{\prime}, \xi_{\mathrm{a}}^{\prime}, t\right)$ for the laser and anti-Stokes modes does not exist. The function $\Phi_{\mathrm{N}}\left(\xi_{1}^{\prime}, t\right)$ is given by equation (4.21) with $\left|\xi_{\mathrm{S}}\right|^{2}=n_{\mathrm{S}}$ and $\left|\xi_{\mathrm{a}}\right|^{2}=n_{\lambda}$.

The quasi-distribution function for the anti-Stokes mode is of the form

$$
\Phi_{\mathrm{N}}\left(\xi_{\mathrm{a}}^{\prime}, t\right)=\left(1 / \pi B_{\mathrm{a}}(t)\right) \exp \left(-\left|\xi_{\mathrm{a}}^{\prime}\right|^{2} / B_{\mathrm{a}}(t)\right)
$$

where the variance $B_{\mathrm{a}}(t)$ is given by equation (5.22) and describes the noise from the spontaneous and stimulated Raman effect. From equation (5.29) we see that the initially chaotic anti-Stokes mode remains chaotic for $t>0$.

\section{Photon-phonon anticorrelation effect}

The existence of anticorrelation between incident and scattered photons obviously poses the question of the existence of anticorrelation between photons and phonons. In what follows, we shall consider the possibility of such anticorrelating. For clarity, the photon-phonon normally ordered characteristic functions will be given only for those modes between which anticorrelation occurs. It can be shown that no anticorrelation exists between Stokes photons and phonons. If the initial total density operator of the 
system is given by equation (4.2), the covariance function between the Stokes photons and phonons is positive:

$$
\begin{aligned}
\left\langle\Delta W_{\mathrm{s}} \Delta W_{\mathrm{p}}\right\rangle= & \left|\mathscr{H}_{\mathrm{s}}\right|^{2} t^{2}\left[n_{\mathrm{p}}^{2}\left(\left|\xi_{1}\right|^{2}-\left|\xi_{\mathrm{s}}\right|^{2}\right)+\left|\xi_{\mathrm{s}}\right|^{2} n_{\mathrm{p}}\left(2\left|\xi_{1}\right|^{2}-1\right)\right. \\
& \left.+\left|\xi_{1}\right|^{2}\left(2\left|\xi_{\mathrm{s}}\right|^{2}+2 n_{\mathrm{p}}+1\right)\right]+t^{2}\left(n_{\mathrm{p}}+1\right)\left(\mathscr{H}_{\mathrm{s}} \mathscr{H}_{\mathrm{a}} \xi_{1}^{2} \xi_{\mathrm{s}}^{*} \xi_{\mathrm{a}}^{*}+\mathrm{CC}\right) .
\end{aligned}
$$

Therefore, in this case, correlation between photons and phonons is obvious.

The phonon variance of intensity is given by

$$
\begin{aligned}
\left\langle\left(\Delta W_{\mathrm{p}}\right)^{2}\right\rangle=n_{\mathrm{p}}^{2} & +2\left|\mathscr{H}_{\mathrm{s}}\right|^{2} t^{2}\left[n_{\mathrm{p}}^{2}\left(\left|\xi_{1}\right|^{2}-\left|\xi_{\mathrm{s}}\right|^{2}\right)+\left|\xi_{1}\right|^{2} n_{\mathrm{p}}\left(\left|\xi_{\mathrm{s}}\right|^{2}+1\right)\right. \\
& +2\left|\mathscr{K}_{\mathrm{a}}\right|^{2} t^{2}\left[n_{\mathrm{p}}^{2}\left(\left|\xi_{\mathrm{a}}\right|^{2}-\left|\xi_{1}\right|^{2}\right)+\left|\xi_{\mathrm{a}}\right|^{2} n_{\mathrm{p}}\left(\left|\xi_{1}\right|^{2}+1\right)\right] \\
& +2 t^{2} n_{\mathrm{p}}\left(\mathscr{H}_{\mathrm{s}} \mathscr{H}_{\mathrm{a}} \xi_{1}^{2} \xi_{\mathrm{S}}^{*} \xi_{\mathrm{a}}^{*}+\mathrm{Cc}\right) .
\end{aligned}
$$

meaning that a bunching of phonons takes place. In the second case, when the initial total density operator of the system is given by equation (4.28), the covariance function between the Stokes photons and phonons is positive as well, namely

$$
\begin{aligned}
\left\langle\Delta W_{\mathrm{S}} \Delta W_{\mathrm{p}}\right\rangle= & \left|\mathscr{H}_{\mathrm{S}}\right|^{2} t^{2}\left[n_{\mathrm{S}}^{2}\left(\left|\xi_{1}\right|^{2}-n_{\mathrm{p}}\right)+n_{\mathrm{p}}^{2}\left(\left|\xi_{1}\right|^{2}-n_{\mathrm{s}}\right)\right. \\
& \left.+n_{\mathrm{S}} n_{\mathrm{p}}\left(2\left|\xi_{1}\right|^{2}-1\right)+\left|\xi_{1}\right|^{2}\left(2 n_{\mathrm{S}}+2 n_{\mathrm{p}}+1\right)\right] .
\end{aligned}
$$

The photon variance of intensity is given by

$$
\begin{aligned}
&\left\langle\left(\Delta W_{\mathrm{p}}\right)^{2}\right\rangle= n_{\mathrm{p}}^{2} \\
&+2\left|\mathscr{H}_{\mathrm{s}}\right|^{2} t^{2}\left[n_{\mathrm{p}}^{2}\left(\left|\xi_{1}\right|^{2}-n_{\mathrm{s}}\right)+\left|\xi_{1}\right|^{2} n_{\mathrm{p}}\left(n_{\mathrm{s}}+1\right)\right] \\
&+2\left|\mathscr{H}_{\mathrm{a}}\right|^{2} t^{2}\left(n_{\mathrm{p}}^{2}\left(n_{\mathrm{a}}-\left|\xi_{1}\right|^{2}\right)+n_{\mathrm{a}} n_{\mathrm{p}}\left(\left|\xi_{1}\right|^{2}+1\right)\right] .
\end{aligned}
$$

To conclude, no photon-phonon anticorrelation occurs between the Stokes photons and phonons in spontaneous and stimulated Raman scattering. One can prove that no anticorrelation occurs between anti-Stokes photons and phonons in spontaneous or stimulated Raman scattering.

We shall now show that anticorrelation between the incident laser photons and phonons occurs in spontaneous Raman scattering. The joint normally ordered quasidistribution function for the laser and phonon mode, when the initial total density operator has the form (4.28), is given by

$C_{\mathrm{N}}\left(\beta_{1}, \beta_{\mathrm{p}}, t\right)$

where

$$
\begin{aligned}
= & \exp \left(\beta_{1} \xi_{1}^{*}(t)-\beta_{1}^{*} \xi_{1}(t)-\left|\beta_{1}\right|^{2} B_{1}(t)-\left|\beta_{\mathrm{p}}\right|^{2} B_{\mathrm{p}} \mid t\right)-\beta_{1}\left|\beta_{\mathrm{p}}\right|^{2} G_{\mathrm{lpp}}^{*}(t) \\
& \left.+\beta_{1}^{*}\left|\beta_{\mathrm{p}}\right|^{2} G_{\mathrm{lpp}}(t)+\left|\beta_{1}\right|^{2}\left|\beta_{\mathrm{p}}\right|^{2} B_{\mathrm{lp}}(t)\right)
\end{aligned}
$$

$$
\begin{aligned}
& \xi_{1}(t)=\left[\xi_{1}-\frac{1}{2}\left|\mathscr{H}_{\mathrm{S}}\right|^{2} t^{2} \xi_{1}\left(n_{\mathrm{S}}+n_{p}+1\right)+\frac{1}{2}\left|\mathscr{H}_{\mathrm{a}}\right|^{2} t^{2} \xi_{1}\left(n_{\mathrm{a}}-n_{\mathrm{p}}\right)\right] \exp \left(-\mathrm{i} \omega_{1} t\right) \\
& B_{1}(t)=\left|\mathscr{H}_{\mathrm{S}}\right|^{2} t^{2} n_{\mathrm{S}} n_{\mathrm{p}}+\left|\mathscr{C}_{\mathrm{a}}\right|^{2} t^{2} n_{\mathrm{a}}\left(n_{\mathrm{p}}+1\right) \\
& B_{\mathrm{p}}(t)=\left\langle\hat{a}_{\mathrm{p}}^{+}(t) \hat{a}_{\mathrm{p}}(t)\right\rangle \\
& =n_{\mathrm{p}}+\left|\mathscr{H}_{\mathrm{S}}\right|^{2} t^{2}\left|\xi_{\mathrm{i}}\right|^{2}\left(n_{\mathrm{p}}+1\right)+\left|\mathscr{H}_{\mathrm{S}}\right|^{2} t^{2} n_{\mathrm{S}}\left(\left|\xi_{\mathrm{i}}\right|^{2}-n_{\mathrm{p}}\right) \\
& +\left|\mathscr{K}_{\mathrm{a}}\right|^{2} t^{2} n_{\mathrm{a}}\left(\left|\xi_{1}\right|^{2}+n_{\mathrm{p}}+1\right)-\left|\mathscr{K}_{\mathrm{a}}\right|^{2} t^{2}\left|\xi_{1}\right|^{2} n_{\mathrm{p}} \\
& G_{\mathrm{lpp}}(t)=\left[-\frac{1}{2}\left(\left|\mathscr{H}_{\mathrm{S}}\right|^{2}+\left|\mathscr{H}_{\mathrm{a}}\right|^{2}\right) t^{2} \xi_{1} n_{\mathrm{p}}\left(n_{\mathrm{p}}+1\right)-\left|\mathscr{H}_{\mathrm{S}}\right|^{2} t^{2} \xi_{1} n_{\mathrm{S}} n_{\mathrm{p}}\right. \\
& \left.-\left|\mathscr{H}_{\mathbf{a}}\right|^{2} t^{2} \xi_{1} n_{\mathrm{a}}\left(n_{\mathrm{p}}+1\right)\right] \exp \left(-\mathrm{i} \omega_{\mathrm{l}} t\right) \\
& B_{\mathrm{lp}}(t)=\left|\mathscr{H}_{\mathrm{S}}\right|^{2} t^{2} n_{\mathrm{p}}^{2} n_{\mathrm{S}}+\left|\mathscr{C}_{\mathrm{a}}\right|^{2} t^{2}\left(n_{\mathrm{p}}+1\right)^{2} n_{\mathrm{a}} \text {. }
\end{aligned}
$$


The covariance function for the incident photons and phonons in spontaneous Raman $\left(n_{\mathrm{S}}=n_{\mathrm{a}}=0\right)$ has the form

$$
\begin{aligned}
\left\langle\Delta W_{1} \Delta W_{\mathrm{p}}\right\rangle & =\left(G_{\mathrm{lpp}}(t) \xi_{1}^{*}(t)+G_{\mathrm{pp}}^{*}(t) \xi_{1}(t)\right)_{n_{\mathrm{s}}=n_{\mathrm{a}}=0} \\
& =-\left(\left|\mathscr{H}_{\mathrm{S}}\right|^{2}+\left.\mathscr{H}_{\mathrm{a}}\right|^{2}\right) t^{2}\left|\xi_{1}\right|^{2} n_{\mathrm{p}}\left(n_{\mathrm{p}}+1\right) .
\end{aligned}
$$

Hence, anticorrelation between the laser photons and phonons is obvious. The existence of an anticorrelation effect (6.11) excludes the existence of a function $\Phi_{\mathrm{N}}\left(\xi_{\mathrm{i}}^{\prime}, \xi_{\mathrm{p}}^{\prime}, t\right)$. The quasi-distribution function for the phonon mode is given by the Gaussian function

$$
\Phi_{\mathrm{N}}\left(\xi_{\mathrm{p}}^{\prime}, t\right)=\left(1 / \pi B_{\mathrm{p}}(t)\right) \exp \left(-\left|\xi_{\mathrm{p}}\right|^{2} / B_{\mathrm{p}}(t)\right)
$$

with the variance $B_{\mathrm{p}}(t)=n_{\mathrm{p}}+\left|\mathscr{H}_{\mathrm{s}}\right|^{2} t^{2}\left|\xi_{1}\right|^{2}\left(n_{\mathrm{p}+} 1\right)-\left|\mathscr{H}_{\mathrm{a}}\right|^{2} t^{2}\left|\xi_{l}\right|^{2} n_{\mathrm{p}}$. From equation (6.12) we see that the initially chaotic phonon mode is chaotic for $t>0$. If initially the phonon mode is in vacuum state, it remains so.

Finally, it should be noted that the complete characteristic function $C_{\mathrm{N}}\left(\beta_{1}, \beta_{\mathrm{S}}, \beta_{\mathrm{a}}, \beta_{\mathrm{p}}, t\right)$ can be calculated using the above method. The results, however, are rather complex.

\section{Conclusion}

A fully quantum mechanical treatment of anticorrelation effects in Raman scattering by phonons is given using the quantum characteristic function technique. Anticorrelation effects are intrinsically quantum mechanical and are not contained in classical theory. Anticorrelations between incident laser photons and scattered photons occur in stimulated Raman scattering only. In spontaneous Raman scattering the incident and scattered photons are not correlated. Anticorrelation between scattered photons and phonons occurs neither in spontaneous nor stimulated Raman scattering. Photonphonon anticorrelation takes place between incident photons and phonons in spontaneous Raman scattering.

\section{References}

Agrawal G P and Mehta C L 1974 J. Phys. A: Math. Nucl. Gen. 7 607-16

Bandilla A and Ritze H H 1976 Opt. Commun. 19 169-71

Carmichael H J and Walls D F 1976 J. Phys. B: Atom. Molec. Phys. 9 L43-46, 1199-219

Chaturvedi S, Drummond P and Walls D F 1977 J. Phys. A. Math. Gen. 10 L187-92

Drummond P D, McNeil K J and Walls D F 1979 Opt. Commun. 2 255-8

von Forester T and Glauber R J 1971 Phys. Rev. A 3 1484-511

Gambini R 1977 Phys. Rev. 15A 1157-68

Glauber R J 1966 Physics of Quantum Electronics (New York: McGraw-Hill)

Kielich S, Kozierowski M and Tanaś R 1978 Coherence and Quantum Optics IV ed L Mandel and E Wolf (New York: Plenum) 511-5

Kimble H J, Dagenais M and Mandel L 1977 Phys. Rev. Lett. 39 691-5

Kozierowski M and Tanaś R 1977 Opt. Commun. 21 229-31

Mehta C L 1967 Phys. Rev. Lett. 18 752-4

Mehta C L and Sudarshan E C G 1965 Phys. Rev. 138 B274-80

Mišta L and Peřina J 1977 Acta Phys. Pol. A52 425-30

Mollow B R and Glauber R J 1967 Phys. Rev. 160 1076-108

Mostowski J and Rzążewski K 1978 Phys. Lett. 66A 275-8 
Peřina J 1972 Coherence of Light (London: Van Nostrand)

Peřinová V and Peřina J 1978 Czech. J. Phys. B28 306-24

Shen Y R 1967 Phys. Rev. 155 921-31

Simaan H D 1975 J. Phys. A: Math. Gen. 8 1620-37

Simaan H D and Loudon R 1975 J. Phys. A: Math. Gen. 8 539-54

Stoler D 1974 Phys. Rev. Lett. 33 1397-400

Walls D F 1970 Z. Phys. 237 224-33

- 1973 J. Phys. A: Math. Nucl. Gen. Phys. 6 496-505

Wang C S 1969 Phys. Rev. 182 482-94

Yuen H P 1976 Phys. Rev. A13 2226-43 